\title{
Why Do Physicians Diagnose Gout in Young Adults with Perfect Kidney Function!!
}

\author{
Abdullah M Nasrat ${ }^{1^{\star}}$, Salwa AM Nasrat ${ }^{2}$, Randa M Nasrat ${ }^{3}$ and Mohammad M Nasrat ${ }^{3}$ \\ ${ }^{1}$ Department of Surgery, Balghsoon Clinic, Jeddah, KSA \\ ${ }^{2}$ Department of Physical Therapy, Cardiac Surgery Academy, Cairo, Egypt \\ ${ }^{3}$ Department of Internal Medicine, Helwan General Hospital, Helwan, Egypt
}

*Corresponding author: Abdullah M Nasrat, Department of Surgery, Balghsoon Clinic, Jeddah, PO Box 5261 KSA-21573, Tel: +966 (012) 667 3645; E-mail: abdullahalnasrat@hotmail.com

Rec date: September 25, 2015 Acc date: October 19, 2015 Pub date: October 23, 2015

Copyright: @ 2015 Nasrat AM, et al. This is an open-access article distributed under the terms of the Creative Commons Attribution License, which permits unrestricted use, distribution, and reproduction in any medium, provided the original author and source are credited.

\begin{abstract}
The study aimed at illustration that detection of high levels of serum uric acid in young adults with perfect kidney function should not be considered final diagnosis of hyperuricemic pre-gouty illness.

Gout is a very old disease which exists for thousands of years with joint swelling, pain or tenderness. Hyperuricema has long been established as the major etiologic factor in gout. Gout has recently become the most common presentation of arthritis in developed countries. Hyperuricemia increases the risk of gout and is also a risk factor of cardiovascular diseases. Hyperuricemia could contribute to diabetes, hypertension and arteriosclerosis due to endothelial dysfunction triggered by vascular wall tissue inflammation because of urate crystals deposition. These reasons are sufficient to render physicians anxious in immediate assessment and treatment of elevated serum uric acid levels. On the contrary, patients hesitate to accept the decision of their pre-gouty illness due to elevation of serum urate particularly if they are young and having perfect renal function.
\end{abstract}

H. pylori could migrate or get forced to migrate to the colon leading to colonic re-absorptive error with excess accumulation of fluids and salts in the body; uric acid could be among these reabsorbed elements giving a picture of elevated serum uric acid level that would have no relation to age of the individual or the integrity of his renal function. Furthermore, gout has been recently considered as one of the auto-inflammatory diseases, hence cytokines are the most common mediators of inflammation; therefore, the role played by the increased mucosal production of inflammatory mediators (cytokines) induced by $H$. pylori is supposed to contribute in the pathogenesis of gout. In this situation, hyperuricemia is not expected to be adequately or successfully improved by traditional urate lowering measures regardless of the age of patient or the state of his kidney function.

Thirty three patients aged between 31-40 years, having normal kidney function and frank history of $H$. pylori dyspepsia were included in the study due to newly discovered elevated levels of serum uric acid regardless of their body built or any associated chronic illness.

Existence of colonic $H$. pylori strains was proved by $H$. pylori fecal antigen test. All patients underwent colon clear employing the potent natural senna purge once and colon care was maintained by vinegar-mixed food therapy for one week.

Serum uric acid levels dropped to normal within 3 days after colon clear in 30 patients while the remaining 3 patients showed the same drop at end of the first week of natural therapy.

On conclusion, Detection of high levels of uric acid in young adults should not be considered final diagnosis of pre-gouty illness unless kidney function is assessed and association of $H$. pylori is excluded by specific tests.

Keywords: Gout; Hyperuricema; H. pylori

\section{Introduction}

Gout is a very old disease which exists for thousands of years with joint swelling, pain or tenderness; the first description of symptoms of gout was found in the Egyptian medical papyri dating to 3000 years BC [1]. Hyperuricema has long been established as the major etiologic factor in gout [2]. Gout has recently become the most common presentation of arthritis in developed countries; however, studies indicate that treatment of gout is still unsatisfactory [3].

As much as hyperuricemia increases the risk of gout, it is also a risk factor of cardiovascular diseases. The relationship between hyperuricemia and cardiovascular disease risk has been also clearly emphasized in literature; elevated serum uric acid was found strongly associated with obesity and hyperlipidemia in both men and women. These findings indicate that attention towards cardiovascular complications should be paid among hyperuricemic patients to the 
extent that lowering the level of serum uric acid might provide a novel target for cardiovascular protection $[4,5]$.

In addition to the inflammatory state triggered by urate crystal deposition in the joints, hyperuricemia constitutes additional pathophysiologic sequences due to tissue inflammation mainly in the vascular wall leading to endothelial dysfunction contributing in turn in the pathogenesis of diabetes, hypertension, arteriosclerosis and chronic heart failure [2]. These reasons made physicians anxious to rush in the immediate assessment and treatment of gout once elevated levels of uric acid were detected.

On the contrary, patients try to hesitate accepting that they are becoming candidates of gouty illness particularly if they are young and their renal function is normal. Severe joint pain combined with no obvious signs of physical trauma or injury caused confusion to patients who try to interpret their symptoms. Self-diagnosis or self-medication, financial and work pressure constitute the main reasons for delayed consultation or reluctance to seek medical attention. Delayed diagnosis after consultation was due to misdiagnosis as per confusion towards attacks in joints other than the first metatarso-phalangeal joint. Resistance to the diagnosis was related to the response of patient's belief about the causes of gout and characteristics of individuals likely to be affected [6].

Although gout is potentially curable with long-term urate lowering therapy; confusion about the details of urate measurement has contributed to suboptimal care of patients [7]. As cytokines are the most important soluble mediators of inflammation; new discoveries has considered gout as an auto-inflammatory disease and included a role of inflammatory cytokines in the pathogenesis of gout allowing further understanding and enabling the use of new therapies for the disease that could reduce patient's resistance to existing procedures [3,8-10].

The association of Helicobacter pylori with disease spread during late decades through immune, inflammatory, toxic or different unknown reasons has been sufficiently reported in literature [11].

\section{Aim}

Demonstration that detection of high levels of serum uric acid in young adults should not be considered final diagnosis of hyperuricemia pre-gouty illness so long kidney function is normal.

\section{Design and Settings}

Prospective study done in Balghsoon Clinics in Jeddah, Saudi Arabia between May 2011 and October 2013.

\section{Patients and Methods}

Thirty three patients aged between 31-40 years, having normal kidney function and frank history of $H$. pylori dyspepsia were included in the study due to newly discovered elevated levels of serum uric acid regardless of their body built or any associated chronic illness.

5 patients were overweight, 3 patients were obese but none of them were having any other illness than hyperuricemia while the remaining patients were average built. Among those patients with average built, 5 patients were diabetic, 7 patients were hypertensive and all of them were inadequately controlled on oral anti-diabetic and antihypertensive medications. Serum uric acid level ranged between
6.5-7.3 mg/dl while existence of colonic $H$. pylori strains was proved by sensitive specific tests ( $H$. pylori fecal antigen test) [11].

All patients underwent colon clear employing the potent natural senna purge once and colon care was maintained by vinegar-mixed food therapy for one week [12].

Patients were free to lead their regular life style except restriction of out-side home meals in order to avoid recurrence of abnormalbehavior H. pylori strains. Eradication of $H$. pylori from the colon was confirmed by the same specific test.

\section{Results}

All patients were found positive for existence of colonic $H$. pylori strains and its eradication from the colon after the natural therapy was confirmed by using $H$. pylori fecal antigen test.

The serum uric acid levels dropped below $4 \mathrm{mg} / \mathrm{dl}$ within 3 days after colon clear in 30 patients while the remaining 3 patients showed the same drop at end of the first week of natural therapy.

Interestingly, all diabetic and hypertensive patients became adequately controlled on their own medications while 6 hypertensive patients were able to quit their medications and maintain normal blood pressure values although they were inadequately controlled in spite of regular follow up of medications and carefulness about their style of life.

Patients were followed up for 12 months and they maintained normal uric acid levels as long as they maintained carefulness about their colonic condition.

\section{Ethical Considerations}

An informed signed consent was taken from all patients; they were made aware about safety of the natural remedies employed for them. They were free to quit the study whenever they like.

\section{Discussion}

Traditional risk factors do not appear fully sufficient to explain the rising figures of chronic disease spread all over the world; in a way that further indicates that traditional measures employed to control disease spread can never be adequate or decisive. The latest decades demonstrated flare up of a lot of medical challenges associated or directly related to $H$. pylori existence [11,12]. Different reports in literature have confirmed the association of cytotoxin-associated gene A (cagA) positive $H$. pylori strains, and emphasized that cagA of $H$. pylori encodes a highly immunogenic and virulence-associated protein; the presence of this virulent gene in the body could affect the clinical outcome in many patients [13]. In addition, gout has been recently considered as one of the auto-inflammatory diseases; and hence cytokines are the most common mediators of inflammation, $[3,8]$ therefore; the increased mucosal production of inflammatory cytokines of $H$. pylori could play an integral role in the pathogenesis of gout regardless of the age of patient or the state of his kidney function $[9,10]$.

H. pylori could migrate or get forced to migrate to the colon under the influence of antibiotic violence leading to accumulation of profuse toxic amounts of ammonia unopposed or buffered by any acidity. Ammonia is smooth muscle tonic and in profuse amounts can cause multiple colonic spasms leading to colonic re-absorptive error with 
Citation: Nasrat AM, Nasrat SAM, Nasrat RM, Nasrat MM (2015) Why Do Physicians Diagnose Gout in Young Adults with Perfect Kidney Function!!. Gen Med (Los Angel) 3: 208. doi:10.4172/2327-5146.1000208

Page 3 of 3

excess accumulation of fluids and salts in the body; [11,12] uric acid could be among these reabsorbed elements giving a picture of elevated serum uric acid level that would have no relation to age of the individual or the integrity of his renal function. Therefore; it is vital to emphasize that urate lowering therapies are not definitive treatment in this situation as they do no treat the pathology and hence are not expected to adequately control the resulting hyperuricemia.

It is interesting to notice the obvious improvement on the clinical condition of chronic illness associated with cases of this study after employment of the natural $H$. pylori eradication therapy. A simple conclusion could directly jump up that it is not necessary that gout is always a risk factor for many diseases but $H$. pylori itself could be mostly the main pathogenic risk of many of the diseases spreading during latest decades like diabetes, hypertension, cardiovascular diseases and of course gout amongst them [11,12,14-16].

It is worthy to mention that antibiotics are seldom effective against extra-gastric $H$. pylori strains [17]. A potent natural purgative is the only measure to eradicate $H$. pylori strains migrated to the colon, otherwise; these $H$. pylori strains would remain in the colon for life $[11,12,16]$.

\section{Conclusion}

Revision of the guidelines of diagnosis and treatment of $H$. pylori and many chronic diseases associated with it may be needed. Detection of high levels of uric acid in young adults should not be considered final diagnosis of pre-gouty illness unless kidney function is assessed and association of $H$. pylori is excluded by specific tests.

\section{Acknowledgements}

The study appreciates the facilities and time allowed by Balghsoon Clinics in Jeddah/Saudi Arabia. The continuous support offered by Abdul-Aziz Al-Sorayai Investment Company (ASIC) in Jeddah/Saudi Arabia, the scientific and emotional support of $\mathrm{Dr}$ Ahmed S. Balghsoon and the continuous brotherhood friendly encouragement of Mr. Abdul-Aziz Al-Sorayai are extremely valued and appreciated.

\section{References}

1. Aluaak T, Aluaak A (2015) [Gout and its manifestations, description and treatment in ancient times]. Cas Lek Cesk 154: 194-195.
2. Gliozzi M, Malara N, Muscoli S (2015) The treatment of hyperuricemia. Int J Cardiol.

3. Cal-Kocikowska J, Nawrocka M, Bogdański P (2015) [Gout - from pathogenesis to treatment - progress in the XXI century]. Pol Merkur Lekarski 38: 354-359.

4. Derosa G, Maffioli P, Sahebkar A (2015) Plasma uric acid concentrations are reduced by fenofibrate: A systematic review and meta-analysis of randomized placebo-controlled trials. Pharmacol Res 102: 63-70.

5. Su P, Hong L, Zhao Y, Sun H, Li L (2015) Relationship Between Hyperuricemia and Cardiovascular Disease Risk Factors in a Chinese Population: A Cross-Sectional Study. Med Sci Monit 21: 2707-2717.

6. Liddle J, Roddy E, Mallen CD, Hider SL, Prinjha S, et al. (2015) Mapping patients' experiences from initial symptoms to gout diagnosis: a qualitative exploration. BMJ Open 5: e008323.

7. Dalbeth N, Winnard D, Gow PJ, Boswell DR, Te Karu L, et al. (2015) Urate testing in gout: why, when and how. N Z Med J 128: 65-68.

8. Marcuzzi A, Piscianz E, Valencic E, Monasta L, Vecchi Brumatti L, et al. (2015) To Extinguish the Fire from Outside the Cell or to Shutdown the Gas Valve Inside? Novel Trends in Anti-Inflammatory Therapies. Int J Mol Sci 16: 21277-21293.

9. Owen DA (2003) Gastritis and carditis. Mod Pathol 16: 325-341.

10. Klausz G, Tiszai A, Lénárt Z, Gyulai Z, Tiszlavicz L, et al. (2004) Helicobacter pylori-induced immunological responses in patients with duodenal ulcer and in patients with cardiomyopathies. Acta Microbiol Immunol Hung 51: 311-320.

11. Farinha P, Gascoyne RD (2005) Helicobacter pylori and MALT lymphoma. Gastroenterology 128: 1579-1605.

12. Nasrat AM (2009) The world misconception and misbehavior towards Helicobacter pylori is leading to major spread of illness. The 7th AntiAging Medicine World Congress, Monte-Carlo, Monaco.

13. Bulut Y, Agacayak A, Karlidag T, Toraman ZA, Yilmaz M (2006) Association of cagA+ Helicobacter pylori with adenotonsillar hypertrophy. Tohoku J Exp Med 209: 229-233.

14. Nasrat SAM, Nasrat RM, Nasrat MM (2015) The dramatic spread of diabetes mellitus worldwide and influence of Helicobacter pylori. General Med J 3: 159-62.

15. Nasrat SAM, Nasrat AM (2015) An alternative approach for the rising challenge of hypertensive illness via Helicobacter pylori eradication. J Cardiol Res 6: 221-225.

16. Nsarat RM, Nasrat MM, Nasrat AM (2015) Improvement of idiopatic cardiomyopathy after colon clear. J Cardiol Res 6: 249-254.

17. Grünberger B, Wöhrer S, Streubel B, Formanek M, Petkov V, et al. (2006) Antibiotic treatment is not effective in patients infected with Helicobacter pylori suffering from extragastric MALT lymphoma. J Clin Oncol 24: 1370-1375. 